\title{
Current Status of Traditional Chinese and Western Medicine Treatment of Diabetic Peripheral Vascular Disease
}

\author{
Rong Li1 ${ }^{1}$ Zongliang Song2*, Qingxiao You ${ }^{1}$ \\ 1Shaanxi University of Traditional Chinese Medicine, Xianyang 712046, Shaanxi Province, China \\ ${ }^{2}$ The Affiliated Hospital of Shaanxi University of Traditional Chinese Medicine, Xianyang 712000, Shaanxi Province, China \\ *Corresponding author: Zongliang Song, 1261857852@qq.com

\begin{abstract}
Diabetic peripheral vascular disease (PVD) is one of the common chronic complications of diabetes. The main clinical manifestations of PVD are numbness and coldness of the limbs, resting pain, intermittent claudication, and other symptoms. The combined treatment involving Chinese and Western medicine for PVD has various clinical methods and definite curative effects. It is worthy of in-depth clinical research and application.
\end{abstract}

Keywords: Diabetic peripheral vascular disease; Traditional Chinese medicine treatment; Western medicine treatment; Integrated traditional Chinese and Western medicine treatment

Publication date: July 2021; Online publication: July 30, 2021

\section{Introduction}

Peripheral vascular disease (PVD) is a chronic complication mainly caused by peripheral large, medium and small arterial atherosclerosis in patients with a long course of diabetes. The clinical manifestations of PVD are dry skin of the lower limbs, numbness and coldness of the limbs, resting pain, intermittent claudication, and other symptoms. It also leads to serious complications, such as diabetic foot necrosis ${ }^{[1]}$. Therefore, therapies for PVD and their effects are getting more and more attention, and the use of the combination of Chinese and Western medicine in clinical setting has been growing, and the effect is also clinically affirmed. In this article, we discuss about the progress of Chinese and Western medicine treatment of PVD.

\section{Pathogenesis}

At present, the understanding of the pathogenesis of PVD in Western medicine is still not completely clear. Studies have shown that ${ }^{[2]}$ it is related to the patient's vascular endothelial function damage, and it is also believed that it may be related to autoimmune function, hemodynamic changes, metabolic disorders, genetic factors, etc. Multifactorial interaction is possible in the pathogenesis of PVD. For the treatment of this disease based on the Western medicine, symptomatic treatments, such as blood sugar control, blood pressure stabilization, and microcirculation improvement are often used, but the therapeutic effect is not satisfactory. Traditional Chinese medicine believes that the course of diabetic disease is long, chronic illness enters the collaterals, blood stasis, collateral stasis, and yin deficiency and $q i$ and yang, eventually resulting in yin and yang deficiency, failure of spleen, kidney and other organs, qi deficiency and blood deficiency, and yin and cold stagnation. Phlegm and dampness stagnation is a general understanding of the pathogenesis of traditional Chinese medicine for diabetic PVD. In modern doctors' understanding of its 
pathogenesis, blood stasis has always played a leading role. Chronic disease can cause blood stasis. Blood stasis is the pathological product of body fluid metabolism disorder of diarrhea. Limbs, veins and collaterals are blocked, and blood cannot reach the limbs, leading to PVD ${ }^{[3]}$.

\section{PVD treatments}

\subsection{Traditional Chinese medicine treatment of PVD}

The internal treatment of traditional Chinese medicine is based on the patient's clinical manifestations and etiology and pathogenesis for syndrome differentiation and treatment. The treatment is mainly to invigorate deficiency, relieve turbidity, and remove blood stasis. Among the various methods, activating blood and removing blood stasis is always the main method throughout the treatment process. According to Peng et al. ${ }^{[4]}$, the use of Huangqi Guizhi Wuwu Decoction to treat PVD has a good clinical effect. Xu et al. ${ }^{[5]}$ believe that turbidity is a pathological factor, among others such as dampness and phlegm, that cannot be ignored in the PVD treatment process. According to the course of the disease and different stages, the $q i$ is wet, the liquid is sputum, and the blood is blood stasis, which is associated with blood stasis in different stages. The treatment includes regulating $q i$ and strengthening spleen, eliminating phlegm and protecting kidney, and replenishing $q i$ and dredging collaterals on the basis of removing stasis.

The external treatment of traditional Chinese medicine has rapid effect, significant curative effect and few side effects. This mode of treatment allows for direct observation of the changes of the disease so that corresponding actions can be executed immediately. Common clinical treatments include acupoint puncture, traditional Chinese medicine stains, traditional Chinese medicine fumigation, traditional Chinese medicine application, acupuncture, cupping, and other forms of treatment, all of which have attained certain progress in PVD treatment.

\subsection{Western medicine treatment of PVD}

At present, Western medicine treatment of PVD mainly focuses on expanding the surrounding blood vessels, improving blood circulation, reducing blood hypercoagulability, inhibiting thrombosis, regulating body fluid metabolism, and increasing local blood nutrient supply. Oral aspirin for anticoagulation has become a common medicine for the prevention and treatment of vascular diseases. Phosphodiesterase inhibitors, such as cilostazol, can inhibit platelet aggregation by increasing the content of intracellular cyclic adenylate, and at the same time, inhibit calcium-induced contraction of smooth muscle cells to exert vasodilation effect. Prostaglandin drugs, such as prostaglandin E1, have strong vasodilation effect and can inhibit platelet aggregation and improve peripheral circulation ${ }^{[6]}$. In addition, gliclazide sustained-release tablets can not only lower blood sugar, but also effectively delay the development of PVD ${ }^{[7]}$. For patients with severe disease and obvious vascular occlusion, intravascular intervention or surgical treatment can be selected.

\subsection{Integrated Chinese and Western medicine treatment of PVD}

At present, controlling blood sugar, improving microcirculation, and regulating $q i$ and blood are common clinical treatment of PVD. In the treatment of PVD, a combination of Western medicine and traditional Chinese medicine is often used, maximizing the strengths of each treatment ${ }^{[2]}$. There are also many clinical treatment programs, including Western medicine combined with Chinese medicine special prescription treatment, for instance, Western medicine combined with Huangqi Guizhi Wuwu Decoction, Western medicine combined with Buyang Huanwu Decoction, Western medicine combined with Jiawei Danggui Sini Decoction. There are also self-prepared prescription treatments combining Western medicine and traditional Chinese medicine, for example, self-prepared Huoxue Tongluo mixture ${ }^{[8]}$; the combined use of patented Chinese medicine and Western medicine is also common in the treatment of PVD. Through the treatment of PVD by oral administration of Western medicine combined with traditional Chinese medicine, 
such as Cobalamin combined with Salvia miltiorrhiza, Chuanxiong, Panax notoginseng, etc. in the treatment of Western medicine combined with traditional Chinese medicine foot bath, the patient can get a better clinical treatment effect and thus, this method is effective as a clinical treatment for PVD.

In addition, ultrasound therapy and far-infrared therapy play an important role in the treatment of PVD, and stem cell transplantation techniques have emerged to be potential treatment. Since each method has its limitations, every method is used in a reasonable and coordinated manner, which is of positive significance for improving the prognosis and quality of life of patients.

\section{Conclusion}

In the treatment of this disease, Chinese medicine that promotes blood circulation and removes blood stasis as well as Western medicine for anticoagulation play very important roles. PVD is accompanied by chronic course of diabetes and long-term hyperglycemia that increases the production of end-products of glycation and oxidative stress, as well as promotes inflammation, blood coagulation, and atherosclerosis. In addition, the sedentary lifestyle and unhealthy dietary structure of modern people make blood circulation more difficult and aggravates blood stasis. Therefore, the method of promoting blood circulation and removing blood stasis is getting more attention in the treatment of diabetic patients. The combination of Chinese and Western medicine can improve the prognosis of patients.

\section{Disclosure statement}

The authors declare that there is no conflict of interest.

\section{References}

[1] Xu Q, Zhang ZH, Ma J, 2013, Application of Turbidity Therapy in the Treatment of Diabetes Pain Peripheral Vascular Disease. Jiangsu Traditional Chinese Medicine, 45(10): 35-36.

[2] Zhao JQ, 2016, The Current Status and Progress of Integrated Traditional Chinese and Western Medicine in the Treatment of Diabetic Peripheral Vascular Disease. Continuing Medical Education, 30(10): 153-155.

[3] $\mathrm{Hu}$ WF, Ye X, 2006, Observation on the Curative Effect of Shenmai Injection Combined with Prostaglandin E4 in the Treatment of Diabetic Lower Extremity Vascular Disease. China Traditional Chinese Medicine Science and Technology, 13(3): 188.

[4] Peng BB, Yan L, 2013, Modified Huangqi Guizhi Wuwu Decoction Treats 40 Cases of Diabetic Peripheral Neurovascular Disease. Yunnan Traditional Chinese Medicine and Materia Medica, 34(1): 27-28.

[5] Xu Q, Zhang ZH, Ma J, 2013, Application of Turbidity Therapy in the Treatment of Diabetic Peripheral Vascular Disease. Jiangsu Traditional Chinese Medicine, 45(10): 35-36.

[6] Kobayashi S, 2014, Pathophysiology, Diagnosis and Treatment of Inter Mittent Claudication in Patients with Lumbar Canal Stenosis. World J Orthop, 5(2): 134-145.

[7] Li YR, Zhao S, Wang HX, et al., 2009, Clinical Study of Gliclazide Sustained-Release Tablets on Improving Vascular Function in Patients with Diabetic Peripheral Vascular Disease. Cliniques, 24(4): 296-299.

[8] Xu HM, Teng T, 2010, Clinical Observation on 30 Cases of Diabetic Peripheral Neuropathy Treated with Integrated Traditional Chinese and Western Medicine. Practical Clinical Integrated Traditional Chinese and Western Medicine, 10(3): 24-26. 